\title{
On the Use of the K-Chart for Phase II Monitoring of Simple Linear Profiles
}

\author{
Walid Gani' ${ }^{1}$ and Mohamed Limam ${ }^{2}$ \\ ${ }^{1}$ LARODEC, ISG, University of Tunis, 41 Avenue de la Liberté, 2000 Le Bardo, Tunisia \\ ${ }^{2}$ Dhofar University, P.O. Box 2509, 211 Salalah, Oman \\ Correspondence should be addressed to Walid Gani; walid.gani@cct.gov.tn
}

Received 19 December 2012; Revised 29 March 2013; Accepted 3 April 2013

Academic Editor: Suk joo Bae

Copyright (C) 2013 W. Gani and M. Limam. This is an open access article distributed under the Creative Commons Attribution License, which permits unrestricted use, distribution, and reproduction in any medium, provided the original work is properly cited.

\begin{abstract}
Control charts for monitoring linear profiles are used to control quality processes which are characterized by a relationship between a response variable and one or more explanatory variables. In the literature, the majority of control charts deal with phase II analysis of linear profiles, where the objective is to assess the performance of control charts in detecting shifts in the parameters of linear profiles. Recently, the kernel distance-based multivariate control chart, also known as the K-chart, has received much attention as a promising nonparametric control chart with high sensitivity to small shifts in the process. Despite its numerous advantages, no work has proposed the use of the K-chart for monitoring simple linear profiles and that serves the motivation for this paper. This paper proposes the use of the K-chart for monitoring simple linear profiles. A benchmark example is used to show the construction methodology of the K-chart for simultaneously monitoring the slope and intercept of linear profile. In addition, performance of the K-chart in detecting out-of-control profiles is assessed and compared with traditional control charts. Results demonstrate that the K-chart performs better than the $T^{2}$ control chart, EWMA control chart, and R-chart under small shift in the slope.
\end{abstract}

\section{Introduction}

In the last decade, control charts for monitoring linear profiles have acquired a prominent role in controlling quality processes characterized by a relationship between a response variable and one or more explanatory variables. A control chart for monitoring linear profiles consists of two phases. In phase I, the parameters of the regression line are estimated to determine the stability of the process. In phase II, the goal is to detect shifts in the process from the baseline estimated in phase I. In the literature, the majority of control charts deal with the phase II analysis of linear profiles. Kang and Albin [1] proposed a multivariate $T^{2}$ control chart for monitoring both the intercept and the slope, while Kim et al. [2] suggested the use of three univariate exponentially weighted moving average $\left(\mathrm{EWMA}_{3}\right)$ control charts for simultaneously monitoring the intercept, slope and standard deviation. Zou et al. [3] proposed a multivariate EWMA scheme when the quality process is characterized by a general linear profile. Zhang et al. [4] developed a control chart based on EWMA and
Likelihood ratio test. Zou and Qiu [5] developed the LASSObased EWMA control chart, for monitoring multiple linear profiles. Li and Wang [6] established an EWMA scheme with variable sampling intervals for monitoring linear profiles.

Recently, the kernel distance-based multivariate control chart, also known as the K-chart, developed by Sun and Tsung [7], has received significant attention as a promising nonparametric control chart with high sensitivity to small shifts in the process mean. According to Gani et al. [8], the $\mathrm{K}$-chart gives the minimum volume closed spherical boundary around the in-control process data. It measures the distance between the kernel center and the incoming new sample to be monitored, which can be calculated using support vectors (SVs). The K-chart relies on support vector data description (SVDD) method, developed by Tax and Duin [9], to determine the shape of the sphere. Any point outside the sphere is considered as out-of-control. When monitoring more than two variables, the K-chart uses kernel methods that provide the advantage of dealing with highdimensional data. Several works dealt with the K-chart. 
Kumar et al. [10] suggested an improvement of the K-chart performance by solving the problem of overfitting due to the existence of outliers in data sets. Camci et al. [11] proposed a robust K-chart that can learn from out-of-control samples and developed an effective heuristic for optimizing the kernel parameters of SVDD. Gani et al. [8] provided an assessment of the K-chart by applying it to a real industrial process and showed that the K-chart is more sensitive to small shifts in mean vector than the $T^{2}$ control chart. Furthermore, Gani and Limam [12] provided the MATLAB code for the implementation of the K-chart. Unlike traditional control charts, the $\mathrm{K}$-chart does not require any assumption about the model distribution of quality characteristics and it has the ability to construct flexible control limits based on SVs. All these features serve as incentives to the application of the Kchart for monitoring linear profiles.

In this paper, we propose the use of the K-chart for monitoring simple linear profiles. We show how to construct the K-chart for simultaneously monitoring the slope and intercept of linear profiles. A comparison between the Kchart and traditional control charts, mainly the $T^{2}$ control chart, the EWMA control chart, and the R-chart using a benchmark simulated data, is also discussed in this paper.

This paper is organized as follows. Principles of monitoring linear profiles, with a special focus on phase II analysis, are presented in Section 2. Theoretical background of adaptation of the K-chart for monitoring simple linear profiles is presented in Section 3. A benchmark simulated data is used in Section 4 to illustrate the application of Kchart for simultaneously monitoring the slope and intercept of linear profiles, with a comparison with traditional control charts. In Section 5, in order to assess the performance of the $\mathrm{K}$-chart in detecting small shifts in the slope, we compare it to the $T^{2}$ and EWMA/R control charts using the average run length criterion. Section 6 provides a conclusion.

\section{Monitoring Simple Linear Profiles}

2.1. The Linear Profile Model. We consider the following simple linear profile model

$$
y_{i j}=\beta_{0 j}+\beta_{1 j} x_{i j}+\epsilon_{i j}, \quad i=1,2, \ldots, n, j=1,2, \ldots, m,
$$

where $y_{i j}$ is the $j$ th measurement, $x_{i j}$ is the value of the explanatory variable corresponding to the $j$ th profile, $\beta_{0 j}$ and $\beta_{1 j}$ are, respectively, the intercept and the slope for profile $j$, and $\epsilon_{i j}$ is the $j$ th random error assumed to be independent and normally distributed with mean zero and variances $\sigma^{2}$.

In phase I, the parameters of the model given in (1) are estimated using the least squares method. The estimated slope for profile $j$, denoted by $b_{1 j}$, is given by

$$
b_{1 j}=\frac{\sum_{i=1}^{n}\left(y_{i j}-\bar{y}_{j}\right)\left(x_{i j}-\bar{x}_{j}\right)}{\sum_{i=1}^{n}\left(x_{i j}-\bar{x}_{j}\right)^{2}}
$$

where $\bar{y}_{j}=\left(\sum_{i=1}^{n} y_{i j}\right) / n$ and $\bar{x}_{j}=\left(\sum_{i=1}^{n} x_{i j}\right) / n$, and the estimated intercept for profile $j$, denoted by $b_{0 j}$, is given by

$$
b_{0 j}=\bar{y}_{j}-b_{1 j} \bar{x}_{j}
$$

The variance of residuals, denoted by $\sigma_{j}^{2}$, is estimated by the $j$ th mean square error $\left(\mathrm{MSE}_{j}\right)$ as follows

$$
\sigma_{j}^{2}=\operatorname{MSE}_{j}=\frac{\sum_{i=1}^{n}\left(y_{i j}-b_{0 j}-b_{1 j} x_{i j}\right)^{2}}{n-2} .
$$

The variance of $b_{1 j}$, denoted by $\sigma_{b_{1 j}}^{2}$, is expressed as follows

$$
\sigma_{b_{1 j}}^{2}=\frac{\sigma^{2}}{\sum_{i=1}^{n}\left(x_{i j}-\bar{x}_{j}\right)^{2}} \text {. }
$$

The variance of $b_{0 j}$, denoted by $\sigma_{b_{0 j}}^{2}$, is expressed as follows

$$
\sigma_{b_{0 j}}^{2}=\sigma^{2}\left(\frac{1}{n}+\frac{\bar{x}_{j}}{\sum_{i=1}^{n}\left(x_{i j}-\bar{x}_{j}\right)^{2}}\right) .
$$

For monitoring simple linear profiles in phase I, several types of control charts have been proposed such as the use of $T^{2}$ control chart (Mestek et al. [13], Stover and Brill [14], and Kang and Albin [1]). Besides, Kim et al. [2] proposed the use of three independent Shewhart control charts for monitoring $b_{0 j}, b_{1 j}$, and $\sigma^{2}$. However, phase II monitoring of linear profiles remains the most important step since it aims to assess the performance of control charts in detecting shifts in the parameters of linear profiles. In the following, we present the main control charts for phase II analysis of linear profiles.

\subsection{Control Charts for Phase II Linear Profile Monitoring.} Mahmoud [15] distinguished between two main categories of control charts for phase II. The omnibus control charts category for monitoring simultaneously the intercept and slope and the individual control charts category for monitoring separately individual regression parameters. This paper focuses on the omnibus category, since our objective is to simultaneously monitor the slope and intercept of linear profiles. The most applied traditional control charts in this category are $T^{2}$ control chart, EWMA control chart, and Rchart.

For monitoring $b_{0}, b_{1}$, and $\sigma^{2}$ in phase II, Kang and Albin [1] recommended the use of the $T^{2}$ control chart for $b_{0 j}$ and $b_{0 j}$. The $T^{2}$ statistics for monitoring the intercept and the slope are given by

$$
T_{j}^{2}=\left(z_{j}-\mu\right)^{T} \Sigma^{-1}\left(z_{j}-\mu\right),
$$

where $z_{j}=\left(b_{0 j}, b_{1 j}\right), \mu=\left(\bar{b}_{0}, \bar{b}_{1}\right), \bar{b}_{0}=\left(\sum_{j=1}^{m} b_{0 j}\right) / m, \bar{b}_{1}=$ $\left(\sum_{j=1}^{m} b_{1 j}\right) / m, \Sigma=\left(\begin{array}{cc}\sigma_{b_{0 j}}^{2} & \sigma_{b_{0 j}, b_{1 j}}^{2} \\ \sigma_{b_{0 j}, b_{1 j}}^{2} & \sigma_{b_{1 j}}^{2}\end{array}\right)$. 
The upper control limit (UCL) for the $T^{2}$ control chart is given by

$$
\mathrm{UCL}=\chi_{2, \alpha}^{2},
$$

where $\chi_{2, \alpha}^{2}$ is the $100(1-\alpha)$ percentile of the chi-squared distribution with 2 degrees of freedom.

In addition, Kang and Albin [1] proposed an EWMA control chart to monitor the average deviation from the incontrol line. The EWMA statistics for monitoring $\sigma^{2}$ are given by

$$
\mathrm{EWMA}_{j}=\theta \bar{e}_{j}+(1-\theta) \mathrm{EWMA}_{j-1},
$$

where $\bar{e}_{j}=\sum_{i=1}^{n}\left(e_{i j} / n_{j}\right)$ is the average deviation for sample $j$, $0<\theta<1$ is a smoothing constant, and $\mathrm{EWMA}_{0}=0$.

The lower control limit (LCL) and the UCL for this EWMA chart are given by

$$
\mathrm{LCL}=-L_{1} \sigma \sqrt{\frac{\theta}{n(2-\theta)}}, \quad \mathrm{UCL}=+L_{1} \sigma \sqrt{\frac{\theta}{n(2-\theta)}},
$$

where $L_{1}>0$ is a constant chosen to give a specified incontrol average run length (ARL).

Also, Kang and Albin [1] suggested the use of an $R$-chart for monitoring the process variation as follows

$$
\mathrm{LCL}=\sigma\left(d_{2}-L_{2} d_{3}\right), \quad \mathrm{UCL}=\sigma\left(d_{2}-L_{2} d_{3}\right),
$$

where $L_{2}>0$ is a constant selected to produce a specified incontrol ARL and $d_{2}$ and $d_{3}$ are constants depending on the sample size $n$.

\section{Monitoring Simple Linear Profiles Using the K-Chart}

We consider $\beta_{j}=\left[b_{0 j}, b_{1 j}\right]$, the vector of the intercept and slope for profile $j$, with $j=1, \ldots, m$. The construction of the K-chart for simultaneously monitoring the slopes and intercepts of linear profiles requires two steps. In the first step, a sphere around the samples of $\beta_{j}$ is constructed using SVDD. The sphere should contain the maximum of $\beta_{j}$ with minimum volume. This is equivalent to solving the following quadratic programming

$$
\text { Minimize } \quad F(R, a)=R^{2}
$$

subject to

$$
\left\|\beta_{j}-a\right\|^{2} \leq R^{2}, \quad j=1,2, \ldots, m,
$$

where $F, a$, and $R$, are respectively, the cost function to minimize, the center, and the radius of the sphere. Equation (13) shows that samples of $\beta_{j}$ having a distance smaller than the radius are considered as targets. To allow the possibility of having outliers in the training set, the distance from $\beta_{j}$ to the center $a$ should not be strictly smaller than $R^{2}$, and larger distances should be penalized. Therefore, we introduce slack variables $\xi_{j} \geq 0$ and the minimization problem becomes

$$
\text { Minimize } \quad F(R, a)=R^{2}+C \sum_{j=1}^{m} \xi_{j},
$$

subject to

$$
\left\|\beta_{j}-a\right\|^{2} \leq R^{2}+\xi_{j},
$$

where $C>0$ is a parameter introduced for the trade-off between the volume of the sphere and the errors.

Equation (15) can be incorporated into (14) by using Lagrange multipliers

$$
\begin{aligned}
L\left(R, a, \alpha_{j}, \eta_{j}, \xi_{j}\right) & \\
= & R^{2}+C \sum_{j=1}^{m} \xi_{j} \\
& \quad-\sum_{j=1}^{m} \alpha_{j}\left[R^{2}+\xi_{j}-\left(\left\|\beta_{j}\right\|^{2}-2 a \cdot \beta_{j}+\|a\|^{2}\right)\right]-\sum_{j=1}^{m} \eta_{j} \xi_{j},
\end{aligned}
$$

with the Lagrange multipliers $\alpha_{j} \geq 0$ and $\eta_{j} \geq 0$; $L$ should be minimized with respect to, $R, a, \xi_{j}$ and maximized with respect to $\alpha_{j}$ and $\eta_{j}$. Setting partial derivatives of $L$, we obtain

$$
\begin{gathered}
\sum_{j=1}^{m} \alpha_{j}=1, \\
a=\sum_{j=1}^{m} \alpha_{j} \beta_{j}, \\
C-\alpha_{j}-\eta_{j}=0 .
\end{gathered}
$$

From (19), $\alpha_{j}=C-\eta_{j}, \alpha_{j} \geq 0$, and $\eta_{j} \geq 0$, then Lagrange multipliers $\eta_{j}$ can be removed and we have

$$
0 \leq \alpha_{j} \leq C .
$$

By substituting (17) and (19) into (16), we have

$$
\text { Maximize } L=\sum_{j=1}^{m} \alpha_{j}\left(\beta_{j} \cdot \beta_{j}\right)-\sum_{j, k=1}^{m} \alpha_{j} \alpha_{k}\left(\beta_{j} \cdot \beta_{k}\right) \text {, }
$$

subject to

$$
0 \leq \alpha_{j} \leq C .
$$

A test sample, denoted by $\beta^{\text {test }}$, is accepted when its distance is smaller or equal to the radius. This is equivalent to

$$
\begin{aligned}
\left(\beta^{\text {test }}-a\right)^{\prime}\left(\beta^{\text {test }}-a\right) & \\
= & \left(\beta^{\text {test' }} \cdot \beta^{\text {test }}\right)-2 \sum_{j=1}^{m} \alpha_{j}\left(\beta^{\text {test }} \cdot \beta_{j}\right) \\
& +\sum_{j, k=1}^{m} \alpha_{j} \alpha_{k}\left(\beta_{j} \cdot \beta_{k}\right) \leq R^{2} .
\end{aligned}
$$


Generally, data is not spherically distributed. To make the method more flexible, the vectors of $\beta_{j}$ are transformed to a higher-dimensional feature space. The inner products in (21) and (23) are substituted by a kernel function $K\left(\beta_{j}, \beta_{k}\right)$. In a higher dimension, the sphere becomes a complex form called "hypersphere." The problem of finding the optimal hypersphere is given by

$$
\text { Maximize } L=\sum_{j=1}^{m} \alpha_{j} K\left(\beta_{j}, \beta_{k}\right)-\sum_{j, k=1}^{m} \alpha_{j} \alpha_{k} K\left(\beta_{j}, \beta_{k}\right),
$$

subject to (23).

A test sample $\beta^{\text {test }}$ is accepted when

$$
\begin{gathered}
K\left(\beta^{\text {test }}, \beta^{\text {test }}\right)-2 \sum_{j=1}^{m} \alpha_{j} K\left(\beta^{\text {test }}, \beta_{j}\right) \\
+\sum_{j, k=1}^{m} \alpha_{j} \alpha_{k} K\left(\beta_{j}, \beta_{k}\right) \leq R^{2} .
\end{gathered}
$$

The second step in the construction of the K-chart consists in determining which samples are SVs by solving the following quadratic programming

$$
\text { Maximize } \sum_{j=1}^{m} \alpha_{j} K\left(\beta_{j}, \beta_{j}\right)-\sum_{j, k=1}^{m} \alpha_{j} \alpha_{k} K\left(\beta_{j}, \beta_{k}\right) \text {, }
$$

subject to

$$
0 \leq \alpha_{j} \leq C
$$

Once the SVs are obtained, the kernel distance (KD) of each sample is computed. For a test sample $\beta^{\text {test }}$, the KD is computed as follows

$$
\begin{aligned}
\mathrm{KD}_{\beta^{\text {test }}}= & \left(K\left(\beta^{\text {test }}, \beta^{\text {test }}\right)-2 \sum_{j \in S}^{m} \alpha_{j} K\left(\beta^{\text {test }}, \beta_{j}\right)\right. \\
& \left.+\sum_{j, k \in S}^{m} \alpha_{j} \alpha_{k} K\left(\beta_{j}, \beta_{k}\right)\right)^{1 / 2},
\end{aligned}
$$

where $S$ is the set of SVs.

The KD of SVs, denoted by $\mathrm{KD}_{\mathrm{SVs}}$, represents the UCL for the K-chart used to monitor a new sample $\beta^{\text {test }}$. This can be illustrated by the following hypothesis test

$$
H_{0}: \mathrm{KD}_{\beta^{\text {test }}} \leq \mathrm{KD}_{\mathrm{SVs}} \text { versus } H_{1}: \mathrm{KD}_{\beta^{\text {test }}}>\mathrm{KD}_{\mathrm{SVs}} \text {. }
$$

Under $H_{0}$ the process is considered as in-control and under $H_{1}$ the process is considered as out-of-control, when sample $\beta^{\text {test }}$ was taken.

\section{Application}

This section is devoted to show how to construct the Kchart for simultaneously monitoring the slope and intercept
TABLE 1: Characteristics of SVDD-based one-class when varying the values of $\gamma$.

\begin{tabular}{lccc}
\hline$\gamma$ & Threshold & SVs & $F_{\text {measure }}$ \\
\hline 0.25 & 0.905 & 17 & 0.947 \\
0.50 & 0.809 & 9 & 0.919 \\
0.75 & 0.705 & 8 & 0.947 \\
1.00 & 0.610 & 7 & 0.889 \\
1.25 & 0.535 & 4 & 0.974 \\
1.50 & 0.469 & 4 & 1.000 \\
1.75 & 0.413 & 3 & 0.919 \\
2.00 & 0.363 & 2 & 0.974 \\
\hline
\end{tabular}

of simple linear profiles. In addition, to show the efficacy of our proposed approach, we compare the performance of the $\mathrm{K}$-chart with that of $T^{2}$ control chart, EWMA control chart, and R-chart in detecting out-of-control (OOC) profiles. In this application, we use the benchmark simulated data of Mahmoud [15], where we consider the following in-control profile model: $Y_{i j}=13+2 X_{i}+\epsilon_{i j}$ (where the $\epsilon_{i j}$ are random errors assumed to be independent and normally distributed with mean zero and variance 1 ), with fixed $X$ values of $-3,-1,1$, and 3 . The simulated data set consists of 29 profiles generated as follows. First, 20 in-control profiles were generated. Then, nine OOC profiles were generated, after shifting the slope from 2.0 to 2.4. Details about the simulated data can be found in Mahmoud [15].

During the training phase, the 20 in-control profiles are used to construct the optimal one class using SVDD algorithm. Then, the nine remainder profiles are used to detect OOC states. For the construction of the optimal SVDDbased one-class, the Gaussian kernel function is used and it is defined as follows

$$
K\left(\beta_{j}, \beta_{k}\right)=\exp \left(-\frac{\left\|\beta_{j}-\beta_{k}\right\|^{2}}{2 \gamma^{2}}\right),
$$

where $\gamma>0$ is the width of the Gaussian kernel that controls the complexity of the SVDD boundary. For the determination of the optimal value of $\gamma$, the $F_{\text {measure }}$ criterion is used and it is defined as follows:

$$
F_{\text {measure }}=\frac{2 \times \text { precision } \times \text { recall }}{\text { precision }+ \text { recall }},
$$

where $0 \leq F_{\text {measure }} \leq 1$, precision $=$ true positive rate/(true positive rate + false positive rate), and recall $=1-$ precision. The optimal value of $\gamma$ corresponds to the highest value of $F_{\text {measure }}$.

All calculations were carried out in MATLAB software. The optimal value of the Gaussian kernel width was found to be $\gamma=1.50$, which corresponds to the highest value of $F_{\text {measure }}$, as shown in Table 1. Some other criteria can be used to verify the construction of the optimal SVDD based oneclass such as graphical representations. It can be seen from Figure 1 that, for $\gamma=0.25$ and 0.5 , the construction of SVDDbased one-class is not possible. It is worth noting that the shape of SVDD-based one-class depends on the parameter 


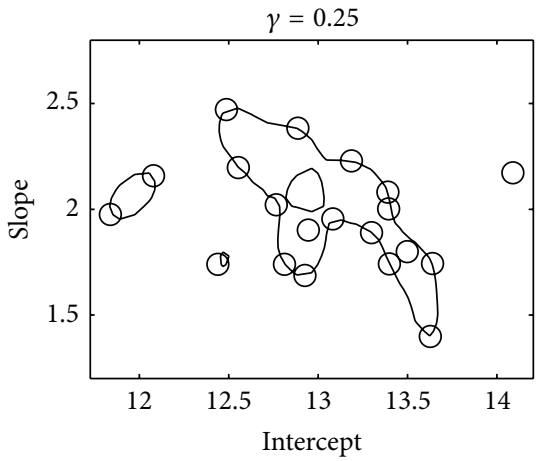

(a)

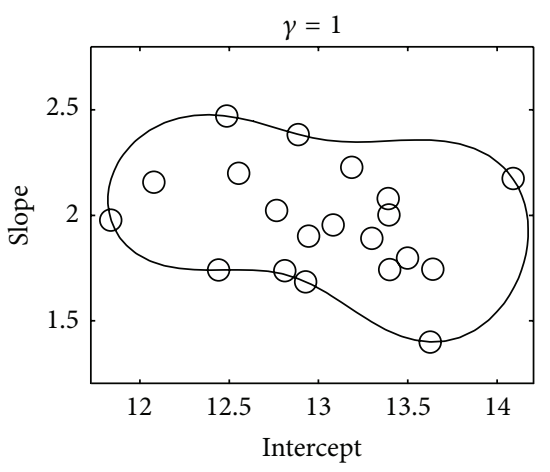

(d)

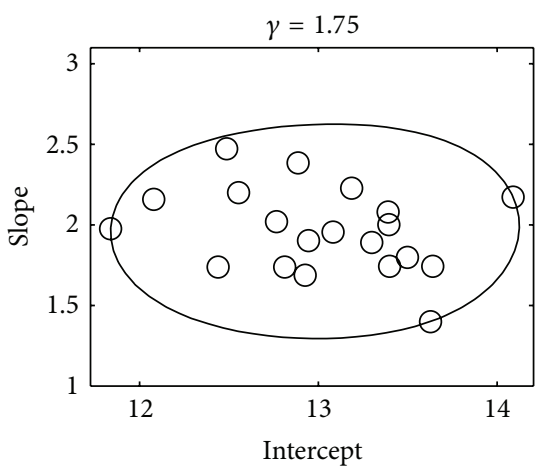

(g)

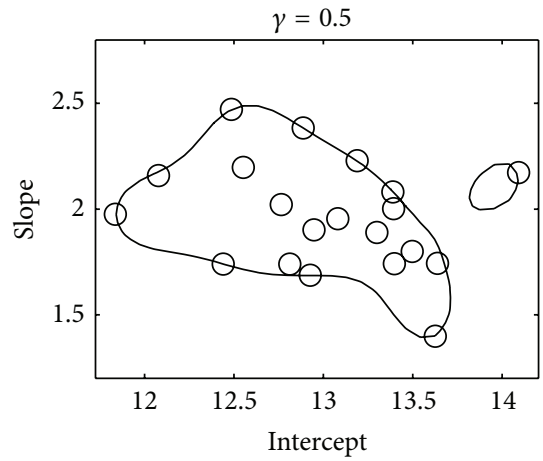

(b)

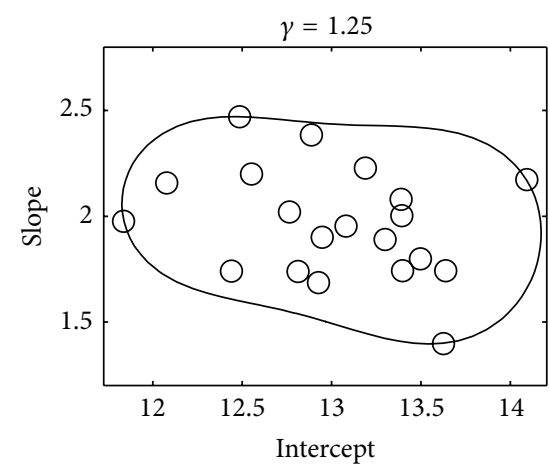

(e)

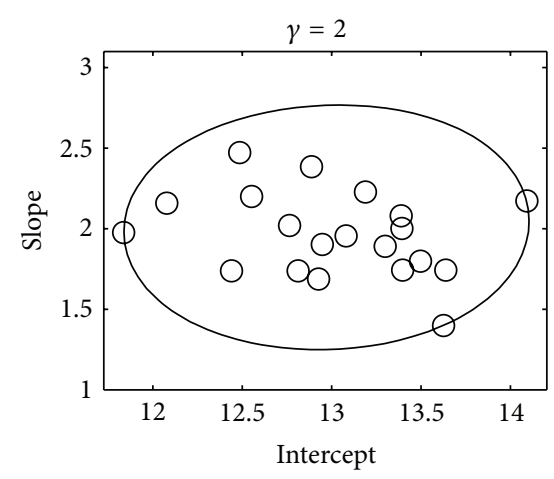

(h)

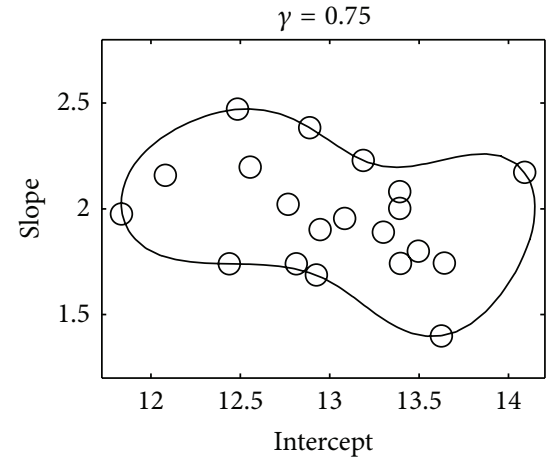

(c)

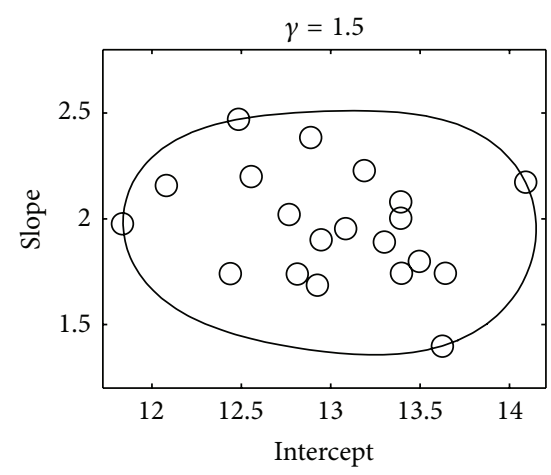

(f)

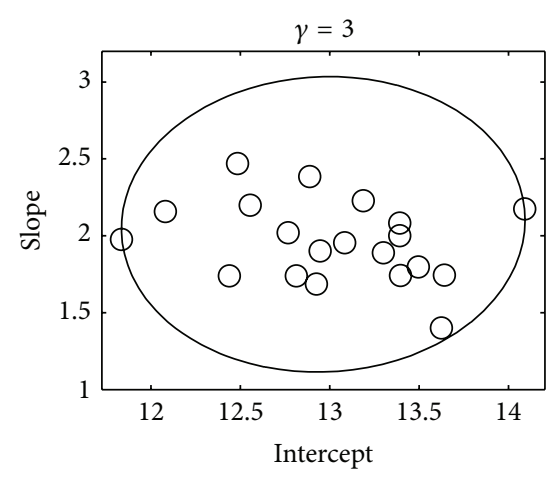

(i)

FIGURE 1: Examples of SVDD-based one-classes for simultaneously monitoring the intercept and slope under different values of the parameter $\gamma$.

$\gamma$. In our application, we stated that the smoothness of the SVDD boundary was enhanced when increasing the value of $\gamma$.

Once the optimal SVDD-based one-class is obtained, the construction of the K-chart is done as follows. After obtaining the solutions of the quadratic programming given by (26) and (27), samples with positive $\alpha_{j}$ are considered as SVs and used to construct the UCL of the K-chart. The latter is based on 4 SVs and it is estimated at 0.469 . The $T^{2}$ control chart was constructed with an UCL $=\chi_{2,0.005}^{2}=10.579$. For the construction of EWMA control chart, the smoothing parameter $\theta$ was set at 0.2 to obtain the charting statistics. Based on (10), the control limits were set at \pm 0.48 so that they produce an in-control ARL of 200. Using (11), the UCL of the R-chart was set at 4.94. It is worth noting that there is no LCL for the R-chart since $n<7$. Figure 2 shows the constructed control charts for phase II.

Regarding the performance of the discussed control charts, the K-chart performed better than the other control charts. In fact, the K-chart detected 6 OOC profiles which are profiles number $23,25,26,27,28$, and 29 , while the $T^{2}$ chart detected only one OOC state which is profile number 27. The R-chart detected one OOC state which is profile number 26. The EWMA control chart was the weakest control chart since it did not detect any OOC profile. As shown in Table 2, the performance rate of K-chart, estimated at $66.67 \%$, was highly superior to that of traditional control charts. The used performance rate in this application is defined as 


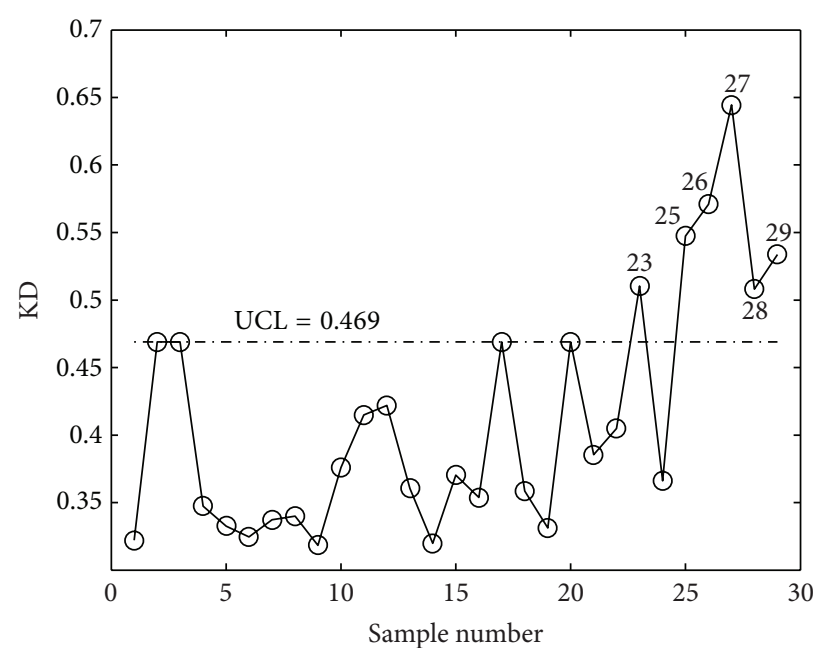

(a)

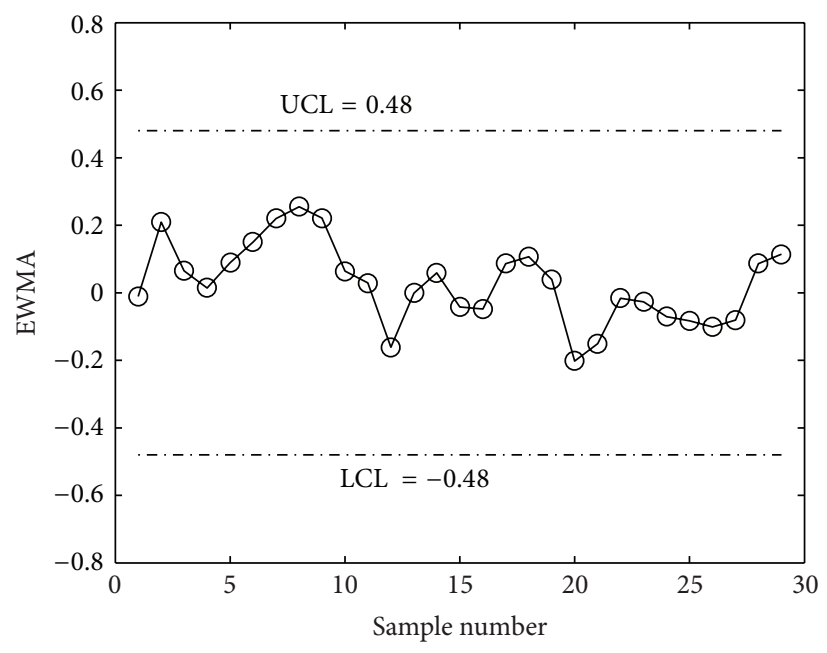

(c)

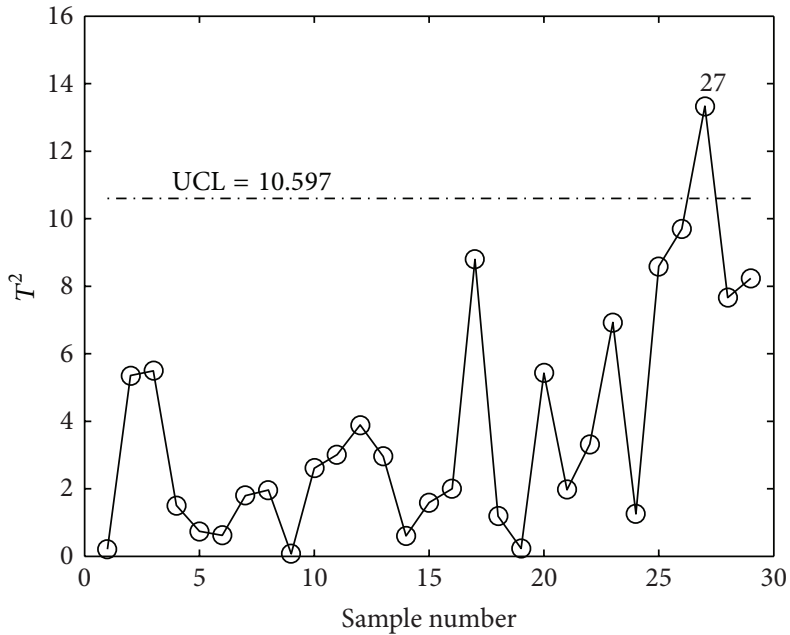

(b)

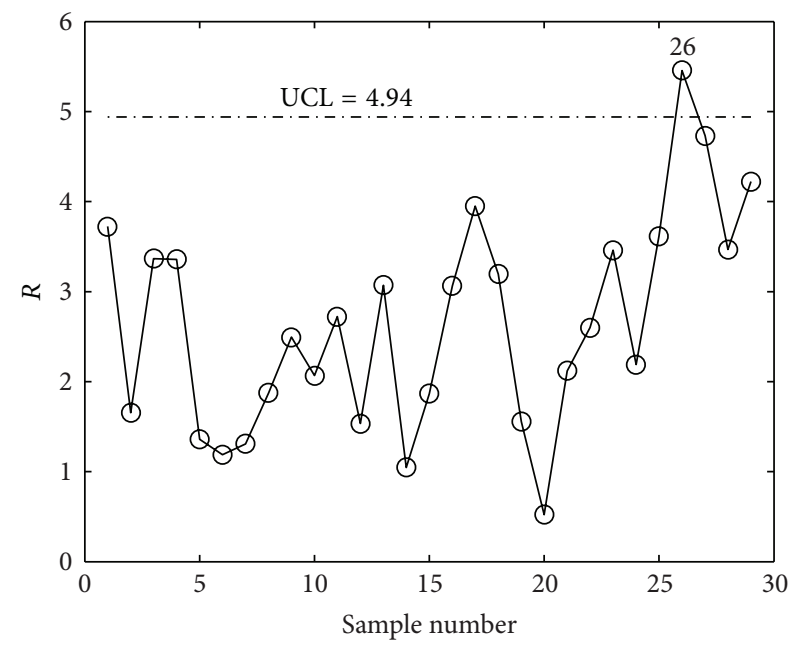

(d)

FIGURE 2: Phase II control charts for monitoring linear profiles: (a) the K-chart, (b) the $T^{2}$ control chart, (c) the EWMA control chart, and (d) the R-chart.

TABLE 2: Performance evaluation of control charts in detecting OOC profiles.

\begin{tabular}{lcc}
\hline Control chart & Number of OOC profiles & Performance rate \\
\hline K-chart & 6 & $66.67 \%$ \\
$T^{2}$ chart & 1 & $11.11 \%$ \\
R-chart & 1 & $11.11 \%$ \\
EWMA chart & 0 & $0.00 \%$ \\
\hline
\end{tabular}

the detected number of OOC profiles divided by the generated number of OOC profiles. A key element that can explain the performance of the $\mathrm{K}$-chart is the nature of its control limit based on SVs, which is very sensitive to any change in kernel's width.

\section{Performance Assessment}

To evaluate the performance of the $\mathrm{K}$-chart in detecting small shifts in the slope, we compare it with the $T^{2}$ and EWMA/R control charts using the ARL criterion. The latter is a common performance measure used to assess the effectiveness of control charts in detecting OOC signal. The ARL is defined as the expected number of samples taken before the shift is detected and it is given by

$$
\mathrm{ARL}=\frac{1}{p},
$$

where $p$ is the probability of one point plots OOC.

For the computation of ARL, a simulation study is conducted based on the model discussed in Section 4 and our results are compared with those reported in Kim et al. [2]. To be consistent with the latter, we follow their methodology which consists in introducing shifts in the slope of linear profiles as follows: $\beta_{1}$ shifts to $\beta_{1}+\delta \sigma$, where $\delta=$ $[-0.2 ;-0.3 ;-0.4 ;-0.5 ;-0.6 ;-0.7 ;-0.8 ;-0.9 ;-1]$. 
TABLE 3: ARL values when $\beta_{1}$ shifts to $\beta_{1}+\delta \sigma$.

\begin{tabular}{lccc}
\hline$\delta$ & $T^{2}$ chart & EWMA/R chart & K-chart \\
\hline 0.00 & 200.000 & 200.000 & 200.000 \\
-0.20 & 52.20 & 76.70 & 14.30 \\
-0.30 & 21.20 & 33.70 & 11.80 \\
-0.40 & 9.60 & 15.30 & 10.50 \\
-0.50 & 4.90 & 7.50 & 9.50 \\
-0.60 & 2.90 & 4.20 & 9.10 \\
-0.70 & 1.90 & 2.60 & 7.70 \\
-0.80 & 1.50 & 1.80 & 7.40 \\
-0.90 & 1.20 & 1.40 & 6.70 \\
-1.00 & 1.10 & 1.20 & 5.40 \\
\hline
\end{tabular}

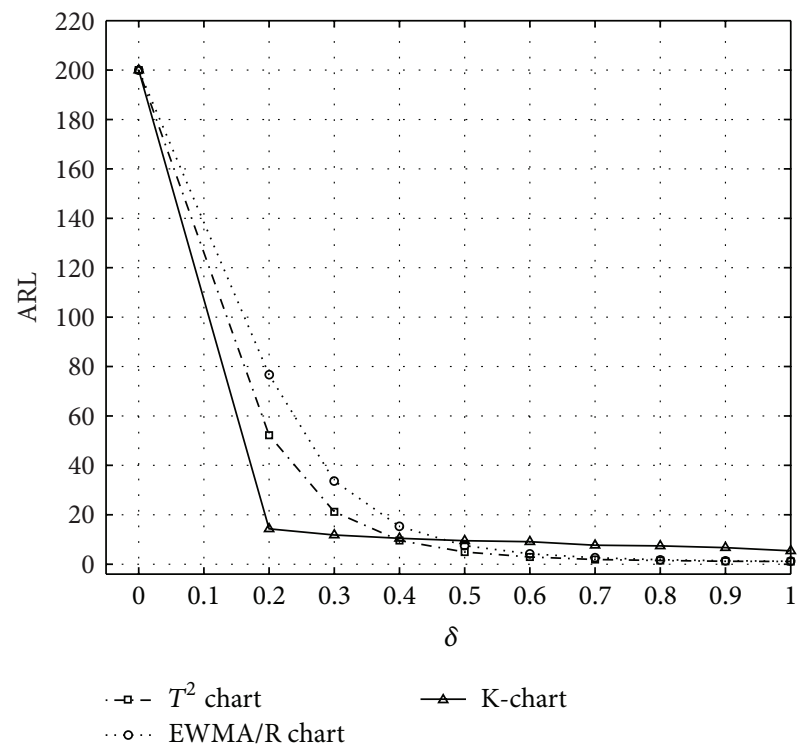

FIGURE 3: ARL comparisons under different values of the shift $\delta$ in the slope.

The K-chart is designed to achieve an overall in-control ARL of 200. The ARL value is estimated by averaging the run lengths obtained by running 10000 simulated charts.

It is clear from Table 3 that the K-chart has better ARL performance under small shifts in the slope. In fact, for $\delta=-0.20$, the $\mathrm{K}$-chart gives an ARL of 14.30, while the $T^{2}$ and EWMA/R control charts give an ARL of 52.20 and 76.70 , respectively. This means that the $\mathrm{K}$-chart requires only 14.30 samples to detect the process shift, while the $T^{2}$ and EWMA/R control charts need 52.20 and 76.70 samples, respectively, to detect the process shift. Figure 3 shows the ARL behavior of the three control charts under different shifts in the slope. It is worth noticing that, for a moderate shift $(\delta=-0.40)$, the $T^{2}$ control chart was slightly better than the K-chart since its ARL was estimated at 9.60 against an ARL of 10.50 for the K-chart. Broadly speaking, from the results of the simulation study one can draw the conclusion that the $\mathrm{K}$-chart is more sensitive to small shifts in the slope of linear profiles than the $T^{2}$ and EWMA/R charts.

\section{Conclusion}

In this paper, we have adapted the $\mathrm{K}$-chart for monitoring simple linear profiles. We show how to construct the Kchart for simultaneously monitoring the slope and intercept. Based on the ARL criterion, the simulation study shows that the K-chart performs better in detecting small shifts in the slope in comparison with $T^{2}$ and EWMA/R control charts. In addition, our application demonstrated that the K-chart is an effective tool for detecting OCC profiles in comparison with traditional control charts. The high sensitivity level of the $\mathrm{K}$-chart is explained by its flexible control limit based on SVs, making it adaptive to any shift in the process. Many interesting extensions are possible for the use of the K-chart for monitoring simple linear profiles. One possible extension is to apply the $\mathrm{K}$-chart for monitoring multivariate linear profiles and compare it with the multivariate EWMA control charts. This extension could constitute a promising research field in the future.

\section{Conflict of Interests}

The authors declare that they do not have a direct financial relation with the software mentioned in this paper and no competing interests.

\section{Acknowledgments}

The authors appreciate the valuable comments of the reviewer which led to a significant improvement of this paper. The authors express their appreciation to LARODEC of ISG, University of Tunis for the support of this work.

\section{References}

[1] L. Kang and S. L. Albin, "On-line monitoring when the process yields a linear profile," Journal of Quality Technology, vol. 32, no. 4, pp. 418-426, 2000.

[2] K. Kim, M. A. Mahmoud, and W. H. Woodall, "On the monitoring of linear profiles," Journal of Quality Technology, vol. 35, no. 3, pp. 317-328, 2003.

[3] C. Zou, F. Tsung, and Z. Wang, "Monitoring general linear profiles using multivariate EWMA schemes," Technometrics, vol. 49, no. 4, pp. 395-408, 2007.

[4] J. Zhang, Z. Li, and Z. Wang, "Control chart based on likelihood ratio for monitoring linear profiles," Computational Statistics and Data Analysis, vol. 53, no. 4, pp. 1440-1448, 2009.

[5] C. Zou and P. Qiu, "Multivariate statistical process control using LASSO," Journal of the American Statistical Association, vol. 104, no. 488, pp. 1586-1596, 2009.

[6] Z. Li and Z. Wang, "An exponentially weighted moving average scheme with variable sampling intervals for monitoring linear profiles," Computers and Industrial Engineering, vol. 59, no. 4, pp. 630-637, 2010.

[7] R. Sun and F. Tsung, "A kernel-distance-based multivariate control chart using support vector methods," International Journal of Production Research, vol. 41, no. 13, pp. 2975-2989, 2003.

[8] W. Gani, H. Taleb, and M. Limam, "An assessment of the kerneldistance-based multivariate control chart through an industrial 
application," Quality and Reliability Engineering International, vol. 27, no. 4, pp. 391-401, 2011.

[9] D. M. J. Tax and R. P. W. Duin, "Support vector data description," Machine Learning, vol. 54, no. 1, pp. 45-66, 2004.

[10] S. Kumar, A. K. Choudhary, M. Kumar, R. Shankar, and M. K. Tiwari, "Kernel distance-based robust support vector methods and its application in developing a robust K-chart," International Journal of Production Research, vol. 44, no. 1, pp. 77-96, 2006.

[11] F. Camci, R. B. Chinnam, and R. D. Ellis, "Robust kernel distance multivariate control chart using support vector principles," International Journal of Production Research, vol. 46, no. 18, pp. 5075-5095, 2008.

[12] W. Gani and M. Limam, "Performance evaluation of oneclass classification-based control charts through an industrial application," Quality and Reliability Engineering International, 2012.

[13] O. Mestek, J. Pavlík, and M. Suchánek, "Multivariate control charts: control charts for calibration curves," Fresenius' Journal of Analytical Chemistry, vol. 350, no. 6, pp. 344-351, 1994.

[14] F. S. Stover and R. V. Brill, "Statistical quality control applied to ion chromatography calibrations," Journal of Chromatography A, vol. 804, no. 1-2, pp. 37-43, 1998.

[15] M. A. Mahmoud, "Simple linear profiles," in Statistical Analysis of Profile Monitoring, R. Noorossana, A. Saghaei, and A. Amiri, Eds., pp. 21-92, John Wiley \& Sons, New York, NY, USA, 2011. 

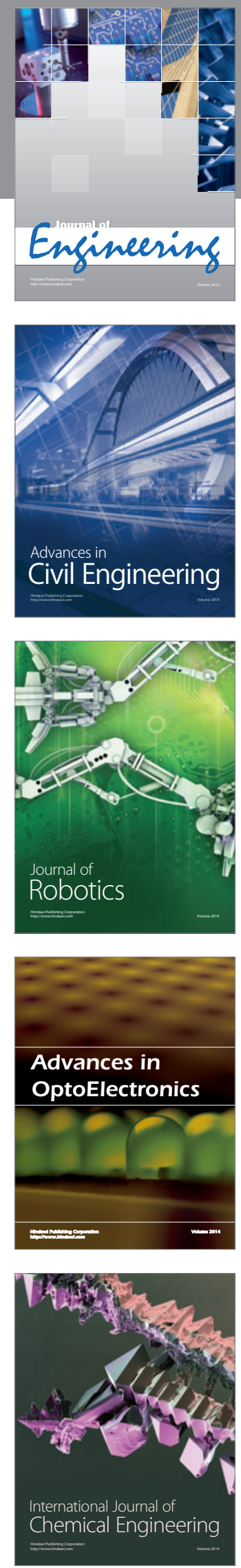

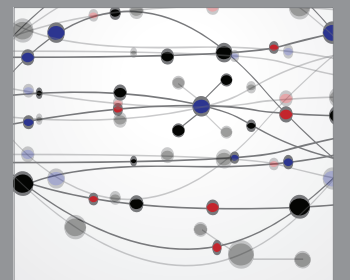

The Scientific World Journal
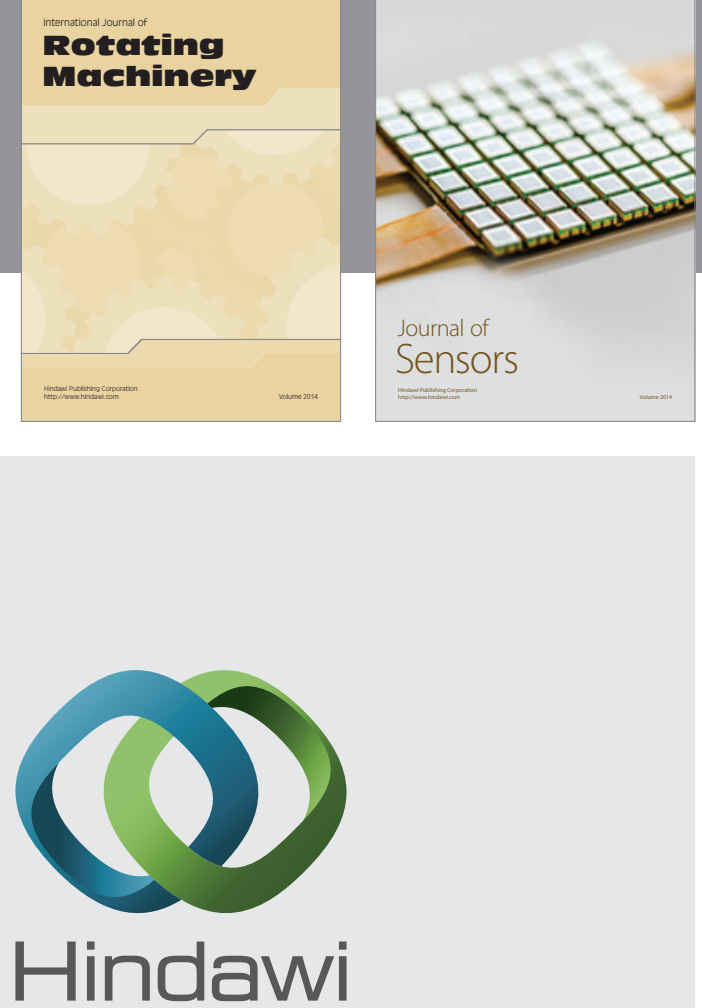

Submit your manuscripts at http://www.hindawi.com
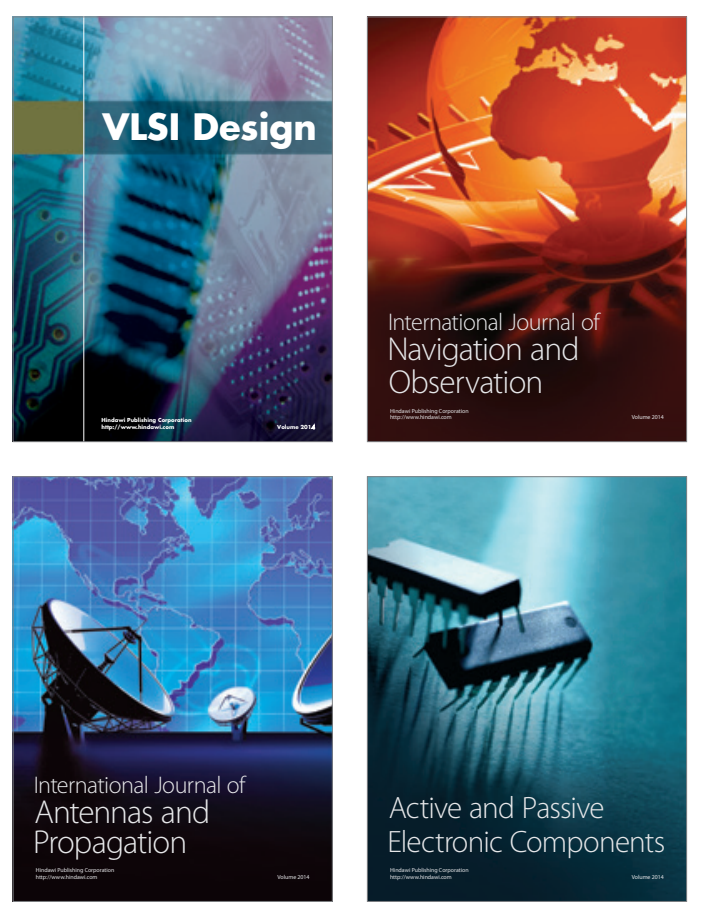
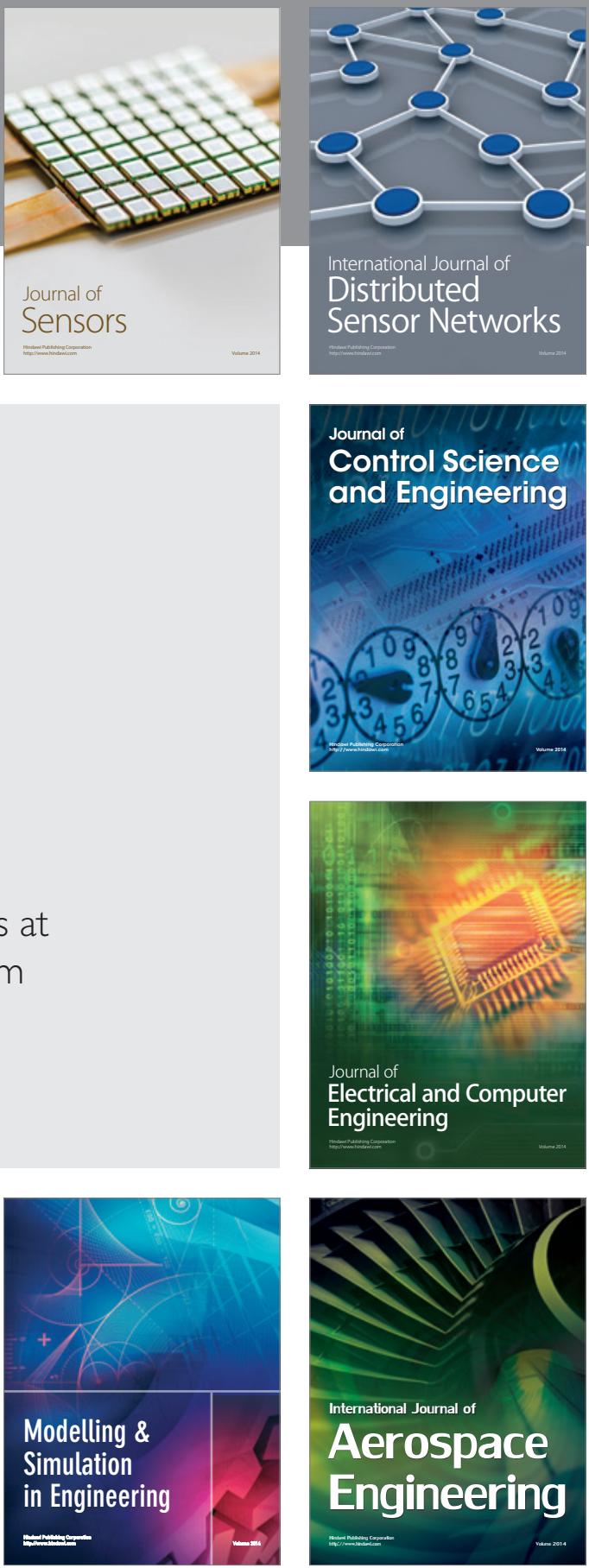

Journal of

Control Science

and Engineering
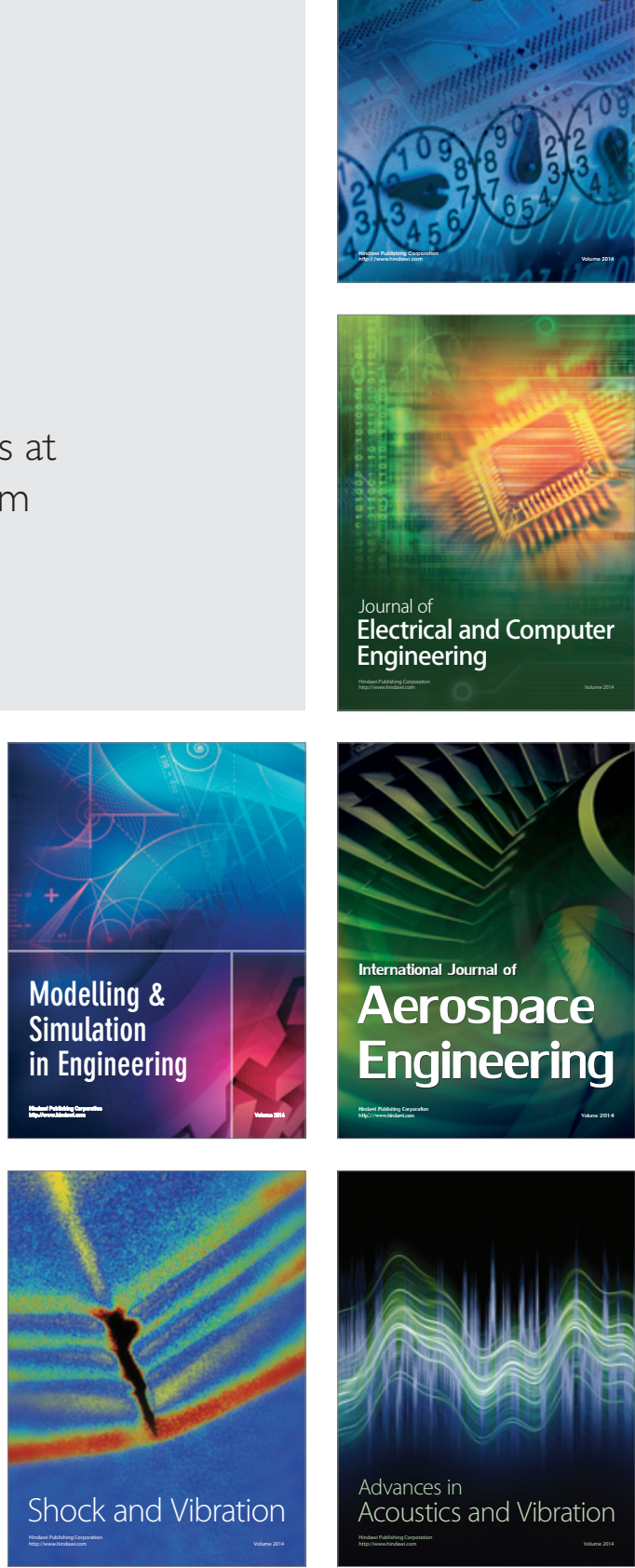